\title{
DISCAPACIDAD INTELECTUAL Y EL USO DE LAS TECNOLOGÍA DE LA INFORMACIÓN Y COMUNICACIÓN: REVISIÓN SISTEMÁTICA
}

\author{
Brizeida Hernández Sánchez \\ Universidad Especializada de las Américas/Panamá \\ hernandez.brizeida@gmail.com \\ Gioconda Vargas Morua \\ Universidad Nacional de Educación a Distancia. Costa Rica \\ gvargasm@uned.ac.cr \\ Greisy González Cedeño \\ Universidad Especializada de las Américas/Panamá \\ greisy3028@gmail.com \\ José Carlos Sánchez García \\ Universidad de Salamanca. España \\ jsanchez@usal.es
}

Recepción Artículo: 24 enero 2020 Admisión Evaluación: 4 marzo 2020

Informe Evaluador 1: 1 enero 2020

Informe Evaluador 2: 1 enero 2020

Aprobación Publicación: 20 abril 2020

\section{RESUMEN}

Este artículo ofrece una visión general existente sobre discapacidad intelectual y su relación con las tecnologías de Información y Comunicación (TIC) y con el objetivo de dar una perspectiva global a través de la revisión sistemática de la literatura qué estudios se han realizado sobre discapacidad intelectual y el uso de las TIC como forma de inclusión, a qué conclusiones se ha llegado, y cuáles son los siguientes pasos a seguir. A fin que los estudiantes con discapacidad tengan acceso a la educación. Además, se analizan algunos datos bibliométrico, para situar esta investigación dentro del gran tema de investigación "educación inclusiva". Los datos se han obtenido a través de una revisión sistemática de la literatura desde 1993 hasta el 2019. El abordaje es cualitativo por cuanto se emplean todos los artículos científicos de SSCI y Scopus que cumplen con las especificaciones requeridas, asociadas a la búsqueda de las variables "intellectual disabilit*", y "intellectual impair*" (discapacidad intelectual), asociadas con "ICT" e "internet" y sus derivaciones. Los resultados revelan la existencia de siete grandes cluster relacionados: síndrome de Down y otras discapacidades, educación, aprendizaje; discapacidad intelectual y sus padres o cuidadores; discapacidad intelectual y comorbilidad o doble afectación de patología.

Palabras clave: discapacidad intelectual; tecnología de la información y comunicación; internet; revisión de la literatura 


\section{DISCAPACIDAD INTELECTUUAL Y EL UִSO DE LAS TECNOLOGÍA DE LA INFORMACIÓN Y COMUNICACIÓN: REVISIÓN SISTEMÁTICA}

\section{ABSTRACT}

Intellectual Disability and the use of Information and Communication Technology: Systematic review. This article offers an existing overview of intellectual disability and its relationship with Information and Communication Technologies (ICT) and with the aim of giving a global perspective through the systematic review of the literature which studies have been carried out on intellectual disability and the use of ICT as a form inclusion, what conclusions have been reached, and what are the next steps to follow. In order for students with disabilities to have access to education. In addition, some bibliometric data is analyzed, to place this research within the great research topic "inclusive education".

The data has been obtained through a systematic review of the literature from 1993 to 2019. The approach is qualitative in that all the scientific articles from SSCl and Scopus that meet the required specifications are used, associated with the search for variables "Intellectual disabilit *", and "intellectual impair *" (intellectual disability), associated with "ICT" and "internet" and their derivations.

The results reveal the existence of seven large related clusters: Down syndrome and other disabilities, education, learning; intellectual disability and their parents or caregivers; intellectual disability and comorbidity or double pathology involvement.

Keywords: intellectual disability; information and communication technology; internet; literature review

\section{INTRODUCCIÓN}

En las últimas dos décadas, la investigación documental sobre discapacidad y tecnologías de la información y la comunicación ha experimentado un crecimiento en términos de volumen, áreas de aplicación, y sus relaciones con las variables de riesgos vulnerables como la discapacidad.

En consecuencia, la producción académica en esta disciplina ha experimentado un avance extraordinario, según la búsqueda selectiva, hemos obtenido referencias bibliográficas de las bases de datos SSCI y Scopus. El propósito de este artículo es revelar las tendencias y áreas más investigadas en veinte años (1993 hasta el 2019), El objetivo es explorar, a través del análisis estadístico de palabras, los temas claves en está temática, y su interrelación de multidisciplinariedad, durante este periodo de tiempo.

Como es común en todos los ámbitos del conocimiento, existe una gran diversidad de publicaciones las áreas los más comunes recogidos en este artículo como: el síndrome de Down y otras discapacidades, educación, aprendizaje y la accesibilidad a los medios de información y comunicación tecnológica. Lo relacionado principalmente con niños con discapacidad intelectual y sus padres o cuidadores. Discapacidad intelectual que es consecuencia de algún trastorno neurobiológico del desarrollo como es el autismo o de algún trastorno del cerebro como la epilepsia. Y finalmente, factores de riesgo y comorbilidad o doble afectación de patología.

Las Tecnologías de la Información y la Comunicación generan nuevas oportunidades para los grupos vulnerables. Entre estas oportunidades se pueden mencionar, al acceso equitativo al mercado laboral en igualdad de oportunidades, desenvolverse, comunicarse y adquirir una mayor independencia y autonomía. El desarrollo sostenible en la Sociedad de la Información se debe reforzar con la investigación y desarrollo de las tecnologías. Los estándares internacionales de accesibilidad a la información y las comunicaciones son exigidos y ya están establecidos (Claro, 20011; UNESCO, 2011; OMS, 2001).

Proyectos basados en tecnología de la información y la comunicación dirigido a la persona con discapacidad intelectual son de alta prioridad (Ferreira, 2018); programas para fortalecer la capacidad digital son inexcusable en estos momentos. La sociedad está viviendo importante proceso de transformación, que ha supuesto la incorporación de las tecnologías de la información y la comunicación (TIC) en todas las áreas y sectores. La sociedad globalizada avanza a gran velocidad, marcada por nuevas formas de inteligencia colectiva y el empleo de la red. Una sociedad que, además, plantea nuevos escenarios y nuevos retos, que supone la brecha digital (Adell, 1998, 2011; Cabero, et al; 2008; Castells, 2004).

Ante tales desafíos, es una necesidad replantearse nuevas prioridades educativas que le proporcione al ciudadano las estrategias necesarias para hacer frente a tales demandas, entre ellas la necesaria adquisición de com- 
petencias relacionadas con los medios digitales para participar de manera activa y funcional en la sociedad actual (UNESCO, 2008; Punie y Brecko, 2014; Ferrari, 2012).

La competencia digital y la ciudadanía digital son requisitos y también exigencia del mercado laboral, (PérezEscoda et, 2016) son competencias y habilidades que nunca antes se habían planteado para la gestión de la información o para la gestión de problemas en la era digital que exige nuevos conocimientos, esto de cara a la persona con discapacidad (Romero-Fría; y Magro Mazo,2016).

Las nuevas demandas del ciudadano digital es estar activo en el proceso de aprendizaje de estas competencias, así como su uso y utilidad de las tecnologías (Pérez-Escoda, 2016). Las tecnologías poseen enormes posibilidades para las personas con discapacidad: 1) disminución de la brecha social, favorecen el aprendizaje accesible, a lo largo de toda la vida; creación de capacidades para ellos, 2) se puede crear un índice digital para la conectividad cuantificable, 3) permiten crear oportunidades equitativas para las personas con discapacidad intelectual, 4) trabajar en entornos digitales accesibles con lectura fácil para las personas con discapacidad intelectual, 5) disminuir barreras hacia el empleo, 6) formar jóvenes con programas de desarrollo innovador basado en tecnologías. 7) Lograr algún avance en el modelo de vida independiente. 8) Aunar esfuerzos con las autoridades gubernamentales para promover la educación inclusiva y accesible, y finalmente, 9) alcanzar cualificación laboral, específicamente la personas discapacidad intelectual.

Las nuevas demandas del ciudadano digital es estar activo en el proceso de aprendizaje de estas competencias, así como su uso y utilidad de las tecnologías (Pérez-Escoda, 2016). En esta era digital la evolución tecnológica ha impactado los diferentes ámbitos del saber (Gisbert, y Esteve, 2016). Hay que hacer énfasis en la alfabetización digital (Ferreira, 2012; Fraser el at. 2010). Es decir, centrarse en por qué, cuándo, quién y para quién entrenarse en tecnología. Estas destrezas permitirán a la persona con discapacidad, contrastar, descubrir y criticar de forma autónoma sus propias competencias de cara al empleo y de cara al desarrollo personal.

Metodología: El enfoque de esta investigación es cualitativa. El diseño de la investigación busca responden las preguntas de investigación que nos hemos planteado. Con respecto al alcance, primero se hace una revisión exploratoria de todos los artículos científicos encontrados y posteriormente, a través del análisis de cluster se establecen las posibles correlaciones que hay entre la información bajo estudio.

\section{Unidades de análisis:}

La población bajo estudio corresponde a todos Ios artículos científicos de SSCI y Scopus sin restricción de tiempo, que incluyen las palabras "discapacidad intelectual" y "tecnología de la información y comunicación" así como sus variaciones, en su título, resumen, palabras claves o tema. El procedimiento de selección para llevar a cabo la revisión sistemática siguió los siguientes pasos:

Se utilizaron técnicas de citación para seleccionar, analizar e interpretar los patrones de citas dentro de la literatura de educación inclusiva y equidad, y el método PRISMA (Liberati et al., 2009; Urrútia \& Bonfill, 2010). Una vez aprobado el protocolo por el revisor, se procede a buscar la información.

En los buscadores de las dos bases de datos (SSCI y Scopus), hemos empleado el siguiente procedimiento sistemático: Elegimos las palabras "intellectual disabilit*" y "intellectual impair" y las asociamos con "Information and communications technology" e "internet". Se utilizan las comillas y el asterisco a fin de que el buscador encontrará todas las posibles opciones de escritura. En este primer paso, se logran identificar 289 documentos de SSCI y 409 documentos de Scopus. La información se extrae sin ninguna restricción en el tiempo, pues se busca analizar las tendencias en la investigación en la inclusión educativa. Posteriormente, comparamos las extracciones para eliminar los artículos duplicados en la misma base (107) y entre las bases de datos (177), así como otros documentos que no estuvieran relacionados o no tuvieran una revisión exhaustiva por pares (147). Revisamos el abstract de cada artículo, para verificar que todos los artículos seleccionados coincidieran con el tema de investigación.

Siguiendo el protocolo, en total con el programa VOSViewer (Van-Eck \& Waltman, 2010) y Excel, analizamos 267 artículos. Nos aseguramos que la calidad de la información fuera óptima y nos permitiría responder de manera asertiva a nuestras preguntas de investigación. 
Tabla1. Criterios de selección y exclusión.

\begin{tabular}{lrr}
\hline \multicolumn{1}{c}{ Titulo del artículo } & Scopus & Wos \\
\hline "intellectual disabilit*" and "Information and com & 38 & 1 \\
"intellectual disabilit*" and "ICT" & 38 & 73 \\
"intellectual disabilit*" and "internet" & 155 & 212 \\
"intellectual impair*" and "Information and comn & 27 & 0 \\
"intellectual impair" and "ICT" & 63 & 0 \\
intellectual impair* and "internet" & 88 & 3 \\
Total documentos encontrados & 409 & $\mathbf{2 8 9}$ \\
Menos repetidos en la misma base & 61 & 46 \\
Menos repetidos entre las bases & 177 & \\
Solo artículos (se eliminan otros documentos ) & 75 & \\
Menos documentos sin relación directa & 72 & \\
\hline Total de artículos para analizar & $\mathbf{2 6 7}$ \\
\hline
\end{tabular}

\section{Técnicas de recolección:}

En la tabla 1, detallamos la identificación, la extracción sistematizada de los artículos, los códigos boléanos que empleamos y la cantidad de artículos que obtuvimos de cada una de las bases. Con el propósito de ser exhaustivo en la búsqueda y ver el efecto a través del tiempo, no se restringió por años. En la parte de elección, indicamos la cantidad de artículos que excluimos por tratarse de otros documentos que no hubieran sido revisados de manera exhaustiva; 0 se encontraren duplicados entres las bases de datos y se eliminaron otros documentos que al hacer la revisión del título o resumen no estuvieran relacionados con el tema. Finalmente, indicamos la cantidad de artículos que analizamos.

\section{Procesamiento de análisis:}

La investigación se llevó a cabo utilizando el software VOSviewer versión 1.6.7 (Van-Eck \& Waltman, 2010) para construir y visualizar mapas bibliométricos a través de la técnica de similitudes, así como para identificar clusters y sus redes de referencia (Van-Eck \& Waltman, 2010; Waltman, Van-Eck, \& Noyons, 2010) y se empleó Mendeley como gestor bibliográfico.

Con VOSviewer (Van-Eck \& Waltman, 2010) se pueden distinguir dos tipos de mapas bibliográficos, uno basado en la distancia y otro en los gráficos. "Los mapas basados en la distancia son mapas en los que la distancia entre dos elementos refleja la fuerza de la relación entre los elementos. Una distancia menor generalmente indica una relación más fuerte" (Van-Eck \& Waltman, 2010), lo que facilita la identificación de grupos de elementos relacionados. Los basados en los gráficos, las líneas se dibujan entre los elementos para indicar las relaciones. Para este estudio, utilizamos mayormente los mapas basados en distancia.

Para el análisis de los datos se plantean dos estrategias. La primera, corresponde al análisis de los clústeres obtenidos con el programa Vosviewer y la segunda, corresponde al análisis bibliométrico de la información. De esta manera, hemos podido estudiar el tema dentro del campo académico y percibir el avance en la investigación desde 1993 hasta el 2019.

\section{RESULTADOS}

Para identificar todos los posibles campos de investigación y las variables que se han asociado a la "discapacidad intelectual" y la "tecnología de la información y comunicación", Ilevamos a cabo un análisis de co-ocurrencia con un mínimo de 2 veces de ocurrencia por palabra, para un total de frecuencia de 481 palabras claves agrupadas en 7 cluster.

De ese modo las variables son agrupadas por el sistema como se observa en el siguiente universo de palabras claves (Figura 1), donde se pueden observar la gran variedad de áreas donde la "discapacidad intelectual" y la "tecnología de la información y comunicación se encuentra relacionada, con: ansiedad, automatismo, atrofia 
cerebral, niños con discapacidad, deficiencia cognitiva; anormalidad eeg, encefalitis, epilepsia, historia familiar, genes, patología; asistencia tecnológica, cuidadores, comportamiento, autismo, discapacidades, sindrome de Down, politicas de cuidado y salud, internet, conocimientos, leyes, medicación, programas de evaluación, soporte social; adolecentes, bullying, protocolos en las clínicas, aprendizaje en linea, motivación, educación especial; autismo, comunicación, programas de computación, recreación, ICT, inclusión social; adultos, paralisis cerebral, genética, población vulnerable; desorden del comportamiento, mutación genética; drogas, factores de riesgo, fatiga, estado anímico, entre otros

Figura 1: Universo de relaciones en la "discapacidad intelectual (DI)" y la "tecnología de la información y comunicación (ICT)"

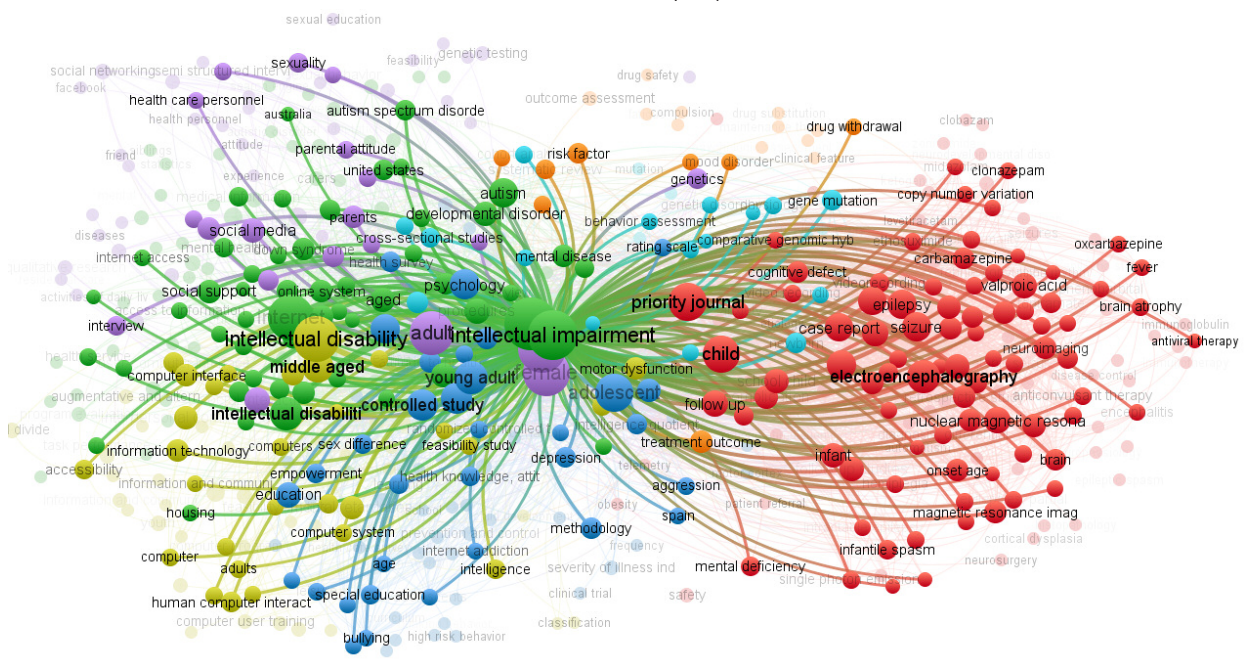

Fuente: propia, a través de VOSviewer

Posteriormente, para identificar los campos de investigación más relevantes, llevamos a cabo un análisis de co-ocurrencia para determinar la mayor frecuencia en el uso de las variables asociadas, de esa manera, se considera un mínimo de 5 veces de ocurrencia por palabra, para un total de frecuencia de 120 palabras claves agrupadas en 4 clúster principales, como se observa en la figura 2. 
Figura 2. Principales relaciones asociadas a "DI" e "ICT"

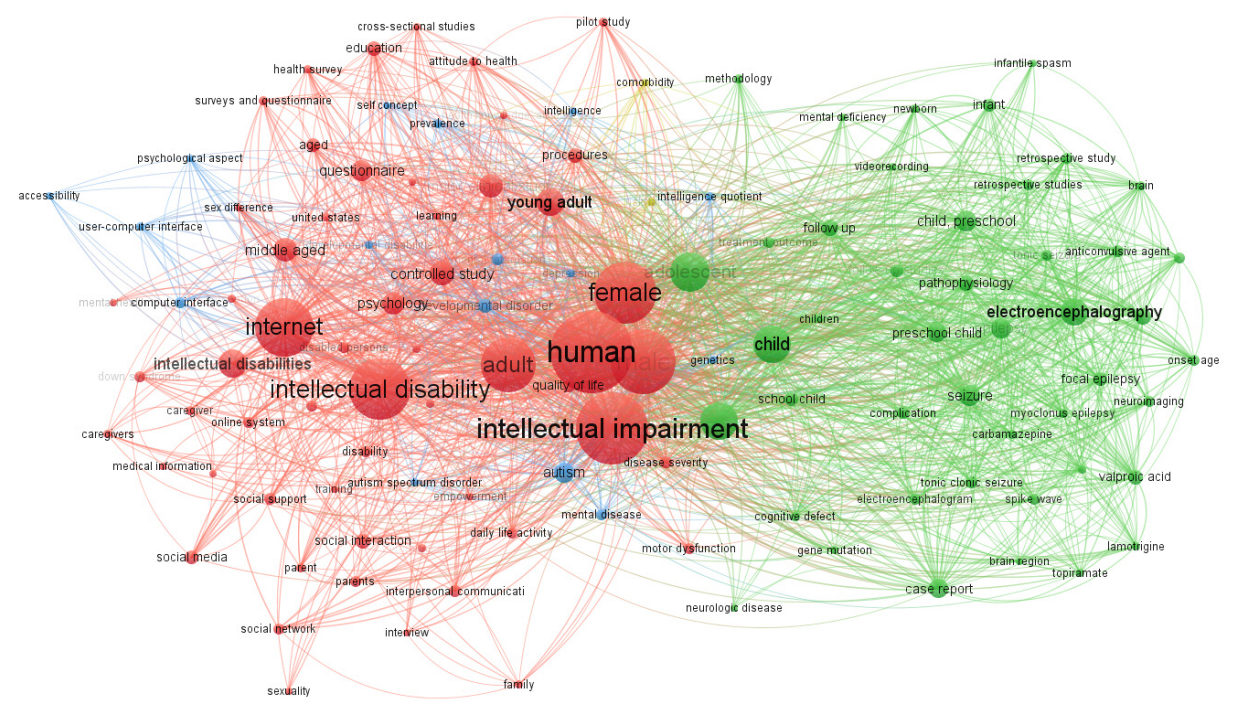

Fuente: propia, a través de VOSviewer

A continuación, se describirán a grandes rasgos las investigaciones asociadas a cada cluster:

El cluster rojo está relacionado con el síndrome de Down y otras discapacidades, educación, aprendizaje y la accesibilidad a los medios de información y comunicación tecnológica. Los estudios relacionados a este clúster revelan que la alfabetización informática de los sujetos con discapacidad intelectual es baja, pues existen dificultades asociadas con la posesión del hardware y software necesarios, un bajo nivel de conocimiento y habilidades de trabajo (Arhipova \& Sergeeva, 2015), dificultades en las habilidades de alfabetización y comunicación del lenguaje cibernético, la etiqueta cibernética y la accesibilidad a equipo adecuado (Caton \& Chapman, 2016). Así como limitaciones potenciales al tratar con fuentes de información no confiables en Internet (Salmerón, Gómez, \& Fajardo, 2016).

Sin embargo, por otro lado también se ha encontrado que algunas personas con discapacidad intelectual con acceso a las ICT están teniendo una actitud positiva, experiencias usando las redes sociales en términos de amistades, desarrollo de identidad social, autoestima y disfrute (Caton \& Chapman, 2016). Pues las redes sociales permiten a las personas con discapacidad intelectual (ID) participar activamente en la sociedad y promover su autodeterminación (Salmerón et al., 2016).

El cluster verde está relacionado principalmente con la relación entre niños con discapacidad intelectual y sus padres o cuidadores. El acceso y el uso de la tecnología de la información y la comunicación (TIC) se está convirtiendo cada vez más en un componente vital de la vida cotidiana, por lo que las investigaciones relacionadas a este clúster exploran en su mayoría los términos de acceso, participación en el aprendizaje y su empleo como medio para mejorar la calidad de vida de las personas con discapacidad. Por tanto, el objetivo del uso de Ias ICT en personas con discapacidad es mejorar la calidad de vida, facilitar la inclusión y el aprendizaje (Meshur, 2016), por ejemplo el uso de dispositivos móviles inteligentes que den soporte en la discapacidad visual o intelectual que posea el usuario (Lancioni et al., 2017).

Por otro lado, también es importante considerar que la cultura organizacional y las creencias sobre la idoneidad de la tecnología para los usuarios de servicios con mayor o menor capacidad, es asumida por el personal 


\section{CRECIMIENTO PSICOLÓGICO Y AFRONTAMIENTO DE LA MADUREZ}

que los atiende (Parsons, Daniels, Porter, \& Robertson, 2008) y que la participación en actividades informáticas en el contexto de comunicación, está determinado por el tipo de apoyo lingüístico brindado por el maestro (Bunning, Heath, \& Minnion, 2010).

El cluster azul se encuentra relacionado con la discapacidad intelectual que es consecuencia de algún trastorno neurobiológico del desarrollo como es el autismo o de algún trastorno del cerebro como la epilepsia. Entre otros aspectos, encontramos en este cluster estudios que se relacionan con la accesibilidad que puede brindar una interfaz, que demuestra la viabilidad de utilizar métodos especiales de diseño de software, como flujos de programas lineales, a fin de minimizar los errores y mejorar el acceso a computadoras de bolsillo para personas con discapacidades intelectuales (Stock, Davies, Davies, \& Wehmeyer, 2006). Otro estudio logró demostrar una mejora general con respecto al desempeño, comprensión de la tarea exigente y percepción sobre el contenido cuando los participantes usaron hipervínculos con imágenes (Rocha et al., 2012).

También en este cluster, se encuentran incluidos estudios que consideran la opinión de los padres con respecto al uso del internet por parte de sus hijos. Los resultados indican que la preocupación de los padres porque sus hijos con discapacidad intelectual se sientan solos al no emplear las redes es mayor, que el riesgo que perciben por el abuso 0 engaño puedan llegar a tener. Consideran más beneficioso el acceso a apoyo y a oportunidades para desarrollar su propia capacidad y experimentar el amor y la sexualidad (Löfgren-Mårtenson, Sorbring, \& Molin, 2015). Los padres tienen la expectativa de que el internet puede ser una herramienta para obtener mayor conciencia sobre su propia discapacidad (Molin, Sorbring, \& Löfgren-Mårtenson, 2015).

El cluster de color amarillo se relaciona principalmente con factores de riesgo y comorbilidad o doble afectación de patología. Por tanto, en este clúster se identifican investigaciones como aquellas que estudian la vulnerabilidad de los jóvenes y su relación con las redes sociales en diferentes fases de la vida adulta y aquellos factores que explican los patrones de adaptación social. Por ejemplo, asistir a clases especiales o tener más de treinta años los aísla en redes pequeñas (Bele \& Kvalsund, 2016). También, el abuso sexual y físico, el aislamiento social, la soledad, la depresión y el chat aumentan el riesgo de ser presa de una solicitud sexual en Internet y se demostró que los jóvenes diagnosticados con una discapacidad intelectual o de desarrollo, son aún más vulnerables a la solicitud sexual en línea (Normand \& Sallafranque-St-Louis, 2016).

Otro aspecto importante de analizar como se observa en la figura 3, es la tendencia en el desarrollo de las investigaciones en "DI" e "ICT". A partir del 2002 inicia un crecimiento en la curva de frecuencia, sin embargo, esta muestra un crecimiento más estable a partir del 2006 y en el 2017 muestra su mayor número de publicaciones en relación al tema. Eso refleja la importancia que ha venido adquiriendo el tema y por ende, el aumento en las investigaciones relacionadas. 
Figura 3. Tendencia en el desarrollo de las investigaciones en "DI" e "ICT".

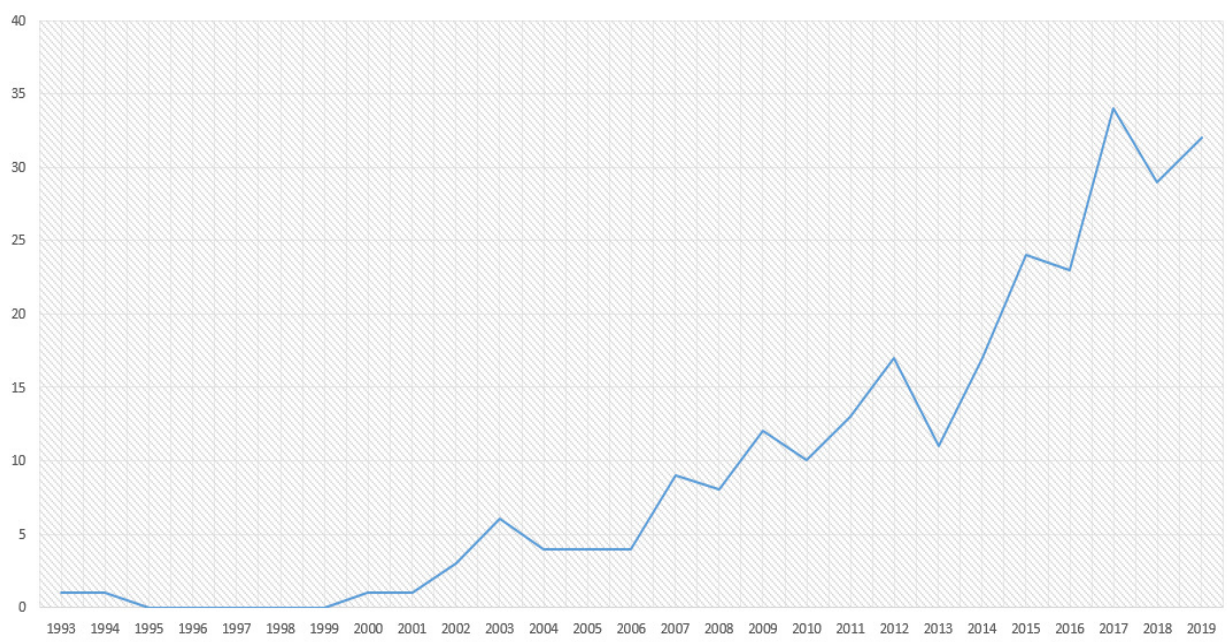

Fuente: propia,

La figura 4 muestra por clúster el desarrollo de las tendencias. Como se puede observar el clúster de color rojo relacionado con el síndrome de Down y otras discapacidades, educación, aprendizaje y la accesibilidad a los medios de información y comunicación tecnológica, así como el de color verde que se relaciona con niños con discapacidad intelectual y sus padres o cuidadores, son los que muestran un crecimiento mayor. Los dos restantes clúster mantienen una constante de poca información.

Figura 4. Tendencia por clúster en el desarrollo de las investigaciones en "DI" e "ICT".

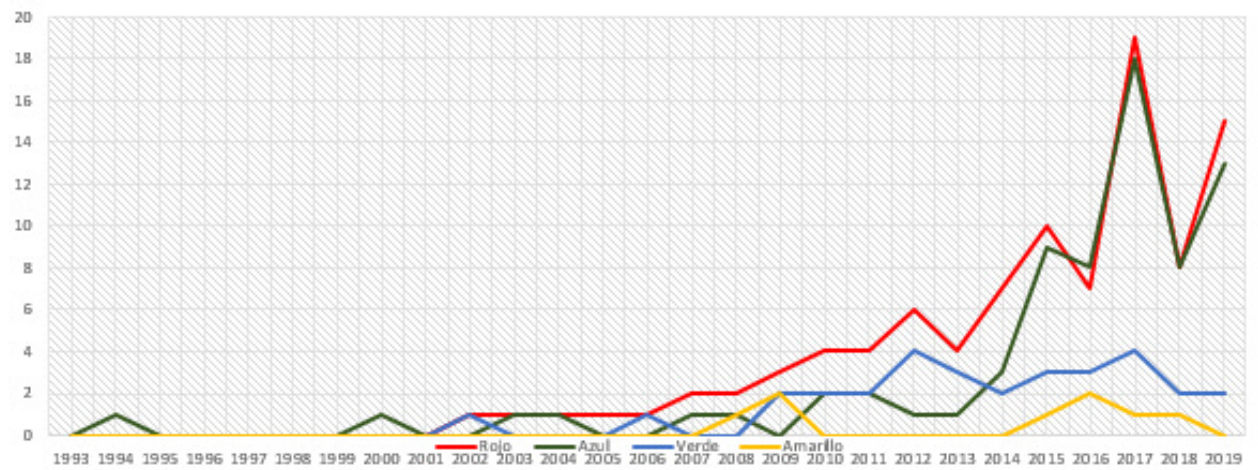

Fuente: propia,

La figura 5 indica cuáles revistas son mayormente citadas en temas relacionados con "DI" e "ICT". Como se puede observar, Cyberpsychology, Journal of applied researchin intellectual disabilitis, Journal of intellectual disability research. 
Figura 5. Revistas que son mayormente citadas en temas de "DI" e "ICT".

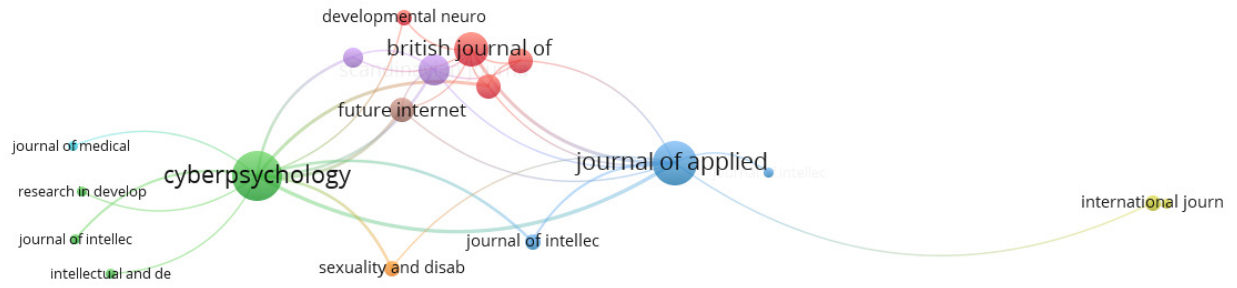

Fuente: propia,

Los autores que mayormente han sido citados por sus publicaciones en el tema son: Normand, Lfgren, Molin, Sorbring y Facardo.

Figura 6. Autores que son mayormente citados en temas de "DI" "e "ICT".

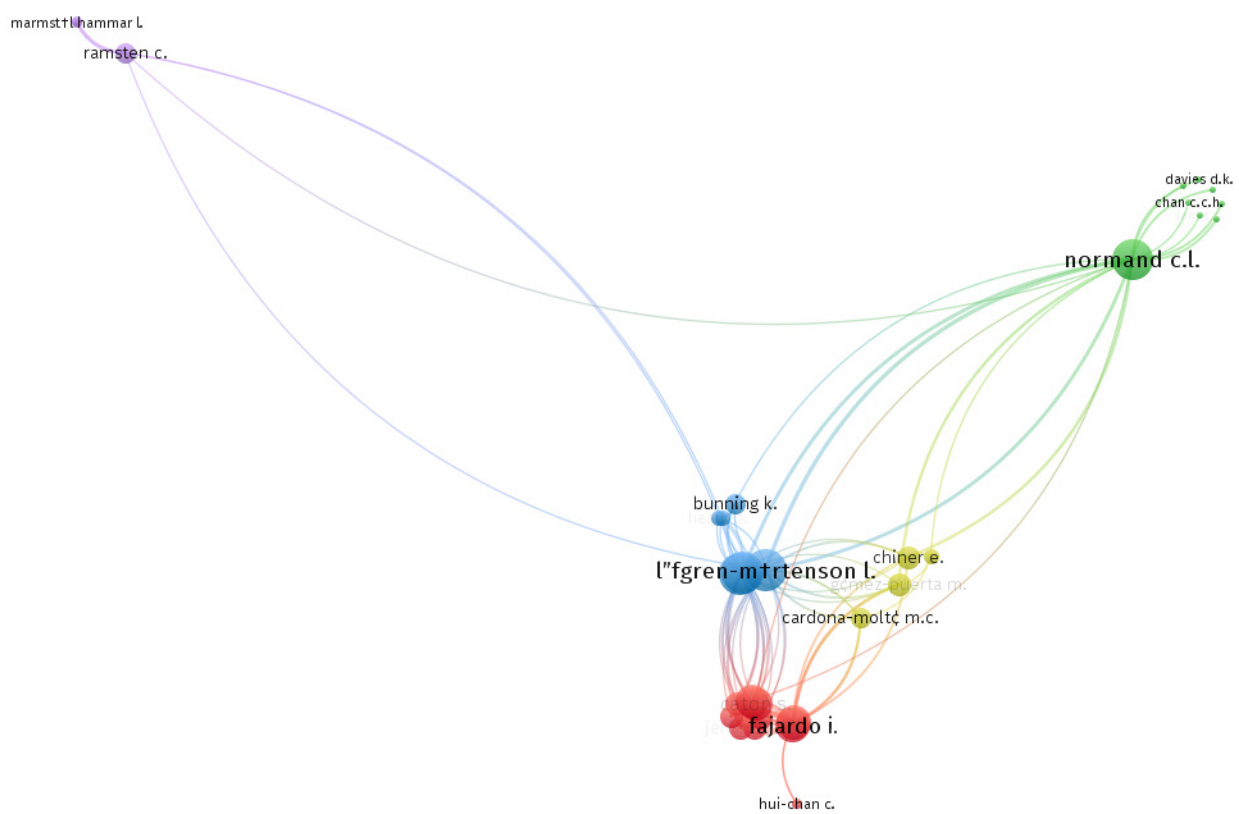

Fuente: propia,

\section{CONCLUSIONES}

En esta investigación ha demostrado cómo han crecido las publicaciones en el campo de la "discapacidad intelectual" y la "tecnologías de la información y la comunicación" en los últimos veinte años, se realizó un análisis de co-concurrencia de 481 palabras claves agrupadas en siete clúster. Se logró identificar los campos de investigación de mayor frecuencia: el síndrome de Down y otras discapacidades, educación, aprendizaje y la accesibilidad a los medios de información y comunicación tecnológica. Lo relacionado principalmente con niños 


\section{DISCAPACIDAD INTELECTUUAL Y EL UUSO DE LAS TECNOLOGÍA DE LA INFORMACIÓN Y COMUNICACIÓN: REVISIÓN SISTEMÁTICA}

con discapacidad intelectual y sus padres o cuidadores. Discapacidad intelectual que es consecuencia de algún trastorno neurobiológico del desarrollo como es el autismo o de algún trastorno del cerebro como la epilepsia. Y los factores de riesgo y comorbilidad o doble afectación de patología.

También se han encontrado que algunas personas con discapacidad intelectual, con acceso a las tecnologías presentan actitud positiva en ámbitos como redes sociales, círculos de amigos, y desarrollo social. Las tecnologías también dan acceso a los aprendizajes, al empleo y contribuyen como medio para mejorar la calidad de vida de estas personas. Otra conclusión a que llegamos es que las familias sienten preocupación por el uso de tecnologías por parte de sus hijos con discapacidad, ya que puede ser objeto de engaños o abusos.

La tendencia en el desarrollo de la investigación muestra un crecimiento estable a partir de 2006 y en el 2017 con mayor frecuencia. Existe un actividad científica constante en la difusión de las tecnologías y la discapacidad por medio de artículos teóricos, que se publican en una amplia variedad de revistas ligados a: Cyberpsychology, Journal of applied researchin intelectual disabilitis, o Journal of intelectual disability research y los autores más destacados entre ellos: Normand, Lfgren, Molin, Sorbring y Facardo.

La convención Internacional sobre el Derecho de las Personas con Discapacidad ONU, (2006) exige políticas que aborden el accesos a las tecnologías accesibles y reclama un monitoreo basado en evidencias para la implementación de cambios de políticas que aborden la accesibilidad y otras inequidades.

En un trabajo futuro, sería útil aplicar técnicas más finas de análisis de conglomerado para examinar los principales campos detectados con mayor detalle, así como para analizar la evolución de las relaciones entre los siete clúster. También sería interesante llevar a cabo una investigación que conecte la tecnología con el empleo en personas con discapacidad intelectual.

Agradecimientos: Este artículo forma parte de las actividades de investigación realizada en el Proyecto ITE18-RI-004 de la Secretaría Nacional de Ciencias, Tecnología e Innovación (SENACYT). Agradecemos al Grupo de Investigación IDEM y al grupo de investigación de la Universidad Estatal a Distancia UNED Costa Rica.

\section{REFERENCIAS BIBLIOGRÁFICAS}

Adell, J. (2011). La competencia digital. Consultado el, 25 de febrero 2018.

Arhipova, S. V., \& Sergeeva, O. S. (2015). Features of the information and communication technology application by the subjects of special education. International Education Studies, 8(6), 162-170. https://doi.org/10.5539/ies.v8n6p162.

Bird, B., y Jelinek, M. (1989). The operation of entrepreneurial intentions. Entrepreneurship theory and practice, 13(2), 21-30.

Bele, I. V., \& Kvalsund, R. (2016). A longitudinal study of social relationships and networks in the transition to and within adulthood for vulnerable young adults at ages 24, 29 and 34 years: compensation, reinforcement or cumulative disadvantages? European Journal of Special Needs Education, 31(3), 314-329. https://doi.org/10.1080/08856257.2016.1187886

Bunning, K., Heath, B., \& Minnion, A. (2010). Interaction between teachers and students with intellectual disability during computer-based activities: The role of human mediation. Technology and Disability, 22(1-2), 61-71. https://doi.org/10.3233/TAD-2010-0278

Cabero, J., Ballesteros, C., Barroso, J., Llorente, M. C., Morales, J. A., Romero, R., \& Román, P. (2008). Aportaciones al e-learning desde la investigación educativa. Sevilla: GID.

Castells, M. (2004). The network society A cross-cultural perspective. Edward Elgar.

Caton, S., \& Chapman, M. (2016). The use of social media and people with intellectual disability: A systematic review and thematic analysis. Journal of Intellectual and Developmental Disability, 41(2), 125-139. https://doi.org/10.3109/13668250.2016.1153052

Claro, M. (2011). El papel de las tecnologías de la información y las comunicaciones en la educación inclusiva.

Ferreira, M. A. (2018). Discapacidad, exclusión social y tecnologías de la información. 
Fraser, B. y Lischer, CJ (2010). Una investigación de la relación entre el rendimiento racial y la tendinitis flexora digital superficial en el caballo de carreras pura sangre. Equine veterinary journal , 42 (4), 322-326.

Gómez-Mejía, L. R., Balkin, D. B., \& Cardy, R. L. (2016). Dirección de recursos humanos. Pearson Educación.

Gisbert, M., \& Esteve, F. (2016). Digital Leaners: la competencia digital de los estudiantes universitarios. La cuestión universitaria, (7), 48-59.

Lancioni, G. E., Singh, N. N., O'Reilly, M. F., Sigafoos, J., Alberti, G., Perilli, V., ... Chiariello, V. (2017). Supporting leisure and communication in people with visual and intellectual disabilities via a smartphonebased program. British Journal of Visual Impairment, 35(3), 257-263. https://doi.org/10.1177/0264619617715497

Liberati, A., Altman, D. G., Tetzlaff, J., Mulrow, C., Gøtzsche, P. C., Ioannidis, J. P. A., ... Moher, D. (2009). The PRISMA statement for reporting systematic reviews and meta-analyses of studies that evaluate health care interventions: explanation and elaboration. Journal of clinical epidemiology (Vol. 62). https://doi.org/10.1016/j.jclinepi.2009.06.006

Löfgren-Mårtenson, L., Sorbring, E., \& Molin, M. (2015). "T@ngled Up in Blue": Views of Parents and Professionals on Internet Use for Sexual Purposes Among Young People with Intellectual Disabilities. Sexuality and Disability, 33(4), 533-544. https://doi.org/10.1007/s11195-015-9415-7

Meshur, H. F. A. (2016). Opportunities for disabled people through ICTs: A perspective from Turkey. International Journal on Disability and Human Development, 15(3), 311-320. https://doi.org/10.1515/ijdhd-2015-0019

Molin, M., Sorbring, E., \& Löfgren-Mårtenson, L. (2015). Teachers' and parents' views on the Internet and social media usage by pupils with intellectual disabilities. Journal of Intellectual Disabilities : JOID, 19(1), 22-33. https://doi.org/10.1177/1744629514563558

Normand, C. L., \& Sallafranque-St-Louis, F. (2016). Cybervictimization of Young People With an Intellectual or Developmental Disability: Risks Specific to Sexual Solicitation. Journal of Applied Research in Intellectual Disabilities, 29(2), 99-110. https://doi.org/10.1111/jar.12163

OMS. (2019). Informe Mundial sobre la Discapacidad promovido por el Banco Mundial.

OMS. (2001), Clasificación Internacional del Funcionamiento, de la Discapacidad y de la Salud (CIF). Organización Mundial de la Salud. Ginebra [en línea. ]http://www.imserso.es/InterPresent2/groups/imserso/documents/.

O'Reilly, A. (2003). El derecho al trabajo decente de las personas con discapacidades. GLADNET Collection, 206.

Parsons, S., Daniels, H., Porter, J., \& Robertson, C. (2008). Resources, staff beliefs and organizational culture: Factors in the use of information and communication technology for adults with intellectual disabilities. Journal of Applied Research in Intellectual Disabilities, 21(1), 19-33. https://doi.org/10.1111/j.14683148.2007.00361.x

Pérez-Escoda, A., Iglesias-Rodríguez, A., y Sánchez-Gómez, M. C. (2016). Nurturing digital citizenship: teachers and students facing digital competences. (pp. 631-636). ACM.

Punie, Y., \& Brecko, B. (2014). Digcomp: Marco europeo de competencias digitales. Bruselas: Comisión Europea. http://jakintza. eus/wp-content/uploads/DIGCOMP_Donosfia_ES-Rev. pdf.

Rocha, T., Bessa, M., Gonçalves, M., Cabral, L., Godinho, F., Peres, E., ... Chalmers, A. (2012). The Recognition of Web Pages' Hyperlinks by People with Intellectual Disabilities: An Evaluation Study. Journal of Applied Research in Intellectual Disabilities, 25(6), 542-552. https://doi.org/10.1111/j.1468-3148.2012.00700.x

Romero-Frías, E., y Magro Mazo, C. (2016). La emancipación intelectual en la sociedad digital: El maestro ignorante de Rancière en nuestros días.

Salmerón, L., Gómez, M., \& Fajardo, I. (2016). How students with intellectual disabilities evaluate recommendations from internet forums. Reading and Writing, 29(8), 1653-1675. https://doi.org/10.1007/s11145-016$9621-4$ 
Stock, S. E., Davies, D. K., Davies, K. R., \& Wehmeyer, M. L. (2006). Evaluation of an application for making palmtop computers accessible to individuals with intellectual disabilities. Journal of Intellectual and Developmental Disability, 31(1), 39-46. https://doi.org/10.1080/13668250500488645

UNESCO. (2011). Informe sobre indicadores o estándares de tecnologías.

Urrútia, G., \& Bonfill, X. (2010). Declaración PRISMA: una propuesta para mejorar la publicación de revisiones sistemáticas y metaanálisis. Medicina Clinica, 135(11), 507-511. https://doi.org/10.1016 /j.medcli.2010.01.015

Van-Eck, N., \& Waltman, L. (2010). Software survey: VOSviewer, a computer program for bibliometric mapping. Scientometrics, 84(2), 523-538. https://doi.org/10.1007/s11192-009-0146-3

Waltman, L., Van-Eck, N., \& Noyons, E. (2010). A unified approach to mapping and clustering of bibliometric networks. Journal of Informetrics, 4(4), 629-635. https://doi.org/10.1016/j.joi.2010.07.002 